\title{
sciendo
}

\section{EFFECTS OF MULBERRY (MORUS ALBA L.) LEAF EXTRACTS ON GROWTH, IMMUNE RESPONSE, AND ANTIOXIDANT FUNCTIONS IN NILE TILAPIA (OREOCHROMIS NILOTICUS)}

\author{
Xuelian Tang ${ }^{1 \sharp}$, Jinghua Fu ${ }^{1 *}$, Qiannan Gao ${ }^{1 \sharp}$, Guangfeng Liu ${ }^{2}$, Jiawei Ye $^{1}$, Wanting Guan ${ }^{1}$, Yifu Shi ${ }^{1}$, \\ Minjun $\mathrm{Xu}^{1,3 \bullet}$ \\ ${ }^{1}$ Laboratory of Guangdong Province and Hong Kong Region on Marine Bioresource Conservation \\ and Exploitation, College of Marine Sciences, South China Agricultural University, Guangzhou, \\ 510642, China \\ ${ }^{2}$ South China Sea Fisheries Research Institute, Guangzhou, 510300, China \\ ${ }^{3}$ Guangdong Modern Agricultural (Quality and Safety of Aquatic Products) Industrial Technology \\ R\&D Center, Guangzhou, 510642, China \\ •Corresponding author: xuminjun@scau.edu.cn, fujinghua@scau.edu.cn \\ \#These authors contributed equally to this work
}

\begin{abstract}
This study evaluates how white mulberry (Morus alba L.) leaf extracts affect the growth, antioxidant activity, and immune response in Nile tilapia Oreochromis niloticus. Mulberry leaf extracts were obtained through aqueous extraction (AE) and ethanol extraction (EE). Powder of mulberry leaf (PML) was added directly to feed and compared with the effects of feeds supplemented with the different extracts. Fish were divided into eight groups for an 8-week feeding trial where they were fed the basal diet or supplemented with 10\% PML, 10\% AE, 20\% AE, 40\% AE, 10\% EE, $\mathbf{2 0 \%}$ EE, or $\mathbf{4 0} \%$ EE. The inclusion of mulberry leaf extract obtained with either method showed better effects on fish growth performance, antioxidant activities and acid phosphatase activity (ACP) in serum, immune cytokine expression, and intestinal morphology as compared with controls or fish fed the $10 \%$ PML diet. The specific growth rate was significantly higher in the $10 \%$ AE, 10\% EE, and 20\% EE groups compared with all other groups $(\mathrm{P}<\mathbf{0 . 0 5})$. Catalase activity was significantly greater in most groups fed an extract, and in the 10\% PML group, when compared with controls. Similarly, ACP, interleukin (IL)-1, and IL-2 expression was significantly increased in groups fed an extract, and in the $10 \%$ PML group, when compared with controls $(P<0.05)$. IL1, IL-2, IL-10, and Toll-like receptor 2 expression was significantly greater in the $10 \% \mathrm{EE}$ group than in the $10 \%$ PML and $10 \%$ AE groups $(\mathrm{P}<0.05)$. Villus length in the middle intestine was significantly increased in the $10 \% \mathrm{AE}$ and $10 \% \mathrm{EE}$ groups compared with controls and the $10 \%$ PML group $(\mathrm{P}<\mathbf{0 . 0 5})$. Thus, $\mathbf{1 0} \%$ mulberry leaf ethanol extract added to feed is recommended for enhancing the growth rate and health of cultured Nile tilapia.
\end{abstract}

Key words: mulberry leaf, Nile tilapia, growth, immune response, antioxidants 
Many factors threaten the health of cultured fish, especially disease outbreaks and environmental pollution. Administration of natural substances as feed additives can enhance the immunity of fish to counteract exposure to various pathogens and toxicants (Mahmoud et al., 2020; Neamat-Allah et al., 2019, 2020, 2021 a).

White mulberry (Morus alba L.) is a fast-growing, small- to medium-sized deciduous tree native to China; it is widely cultivated and has become naturalized in the temperate and tropical regions, including of the Far East and South East Asia, southern Europe, North America, southeastern Australia, and also some parts of Africa (He et al., 2018). Mulberry leaf plays a fundamental role in the traditional silk industry (Chan et al., 2016). Many studies have shown that mulberry leaf has good palatability and is rich in nutrients, with especially high crude protein content and a variety of fatty acids, minerals, vitamins, bioactive substances and phytonutrients (Chan et al., 2016; Chen et al., 2021; Liu et al., 2019). Consequently, mulberry leaf has been successfully used as a dietary protein supplement in aquaculture and in poultry and livestock farming (Ali et al., 2020; Kandylis et al., 2009; Kaviraj et al., 2013; Kong et al., 2019; Lin et al., 2017; Liu et al., 2019; Neamat-Allah et al., 2021 b). Furthermore, mulberry leaf has been suggested as a novel food (Li et al., 2018) and medicinal plant. Assessed in Sprague-Dawley rats, mulberry leaf meal showed no toxic effects on most hematological and coagulation parameters in both males and females (Cai et al., 2019).

Prepared as a traditional Chinese medicine, mulberry leaf has been used for thousands of years, as recorded in the 2015 edition of the Chinese Pharmacopoeia. Pharmacological research has shown that the extracts of mulberry leaf have a range of promising bioactivities, including immunomodulatory, anti-inflammatory, antioxidant, antidiabetic, hepatoprotective and anti-obesity activities (El-Sayyad, 2015; Hao et al., 2018; He et al., 2018; Wang et al., 2012).

The nutritional and medicinal value of mulberry leaf has also been demonstrated in fish. Mulberry leaf has been tested as a potential fishmeal replacement in formulated feed for catfish Heteropneustes fossilis and carp Labeo rohita, and fermented mulberry leaf meal was shown to have excellent nutritional quality for carp (Kaviraj et al., 2013; Mondal et al., 2015). It has been reported that the plant helped alleviate infections with Aeromonas hydrophila in African catfish Clarias gariepinus, (Sheikhlar et al., 2014), had antiparasitic effects against Ichthyophthirius multifiliis in grass carp Ctenopharyngodon idella (Fu et al., 2014; Liang et al., 2015), and mitigated the potential hazards of heavy metals (e.g., cadmium residue) in the liver of the rare minnow Gobiocyprisrarus (Xiong et al., 2020). As a dietary supplement, it also showed inhibitory effects on the production of inflammatory mediators and reactive oxygen species in lipopolysaccharide-stimulated macrophages in zebra fish (Kwon et al., 2017) and antioxidative effects in African catfish (Sheikhlar et al., 2017). Thus, research has confirmed that the mulberry products can be used effectively in aquaculture. Most studies have focused on using the leaves as an alternative source of protein added directly to feed or its use as a fermented blend. However, few studies have reported the effects of mulberry leaf extracts on fish.

We herein investigated the effects of dietary supplementation with mulberry leaf extracts, obtained by either aqueous or ethanol extraction, on the growth, immune 
response and antioxidant functions, meat quality, and intestinal morphology of Nile tilapia Oreochromis niloticus. Mulberry leaf added to a fish feed formulation is ecofriendly and environmentally sustainable; therefore, our results will be beneficial for the further development and application of mulberry leaf in aquaculture.

\section{Material and methods}

\section{Mulberry leaf extraction process}

Mulberry leaves were purchased from a local chemist's shop; the leaves had been picked after the frost period, washed, and left to dry at room temperature. Before extraction in the laboratory, the mulberry leaves were dried further dried at $50^{\circ} \mathrm{C}$ in a blast drying oven (DHG-9140, Haixiang Instrument and Equipment Factory, Shanghai, China) at $50^{\circ} \mathrm{C}$ to a constant weight, and then crushed into a powder, to a size of $<60$ mesh. Finally, two traditional extracting methods, water decoction and ethanol extraction, were used to obtain mulberry leaf extracts from the powder of mulberry leaf (PML).

\section{Water decoction method}

We used the extraction method for Traditional Chinese medicine according to Miao et al. (2019) with slight modification. The PML was first put into a decoction pot and soaked in distilled water for $20 \mathrm{~min}$. Next, it was boiled and simmered for $2 \mathrm{~h}$, and the liquid poured through filter paper. The filtered solids were processed through the same steps three times. Finally, all the filtration liquids were combined and concentrated into $1 \mathrm{~g} / \mathrm{mL}$ crude extract using a rotary evaporator and finally stored at $4{ }^{\circ} \mathrm{C}$ in a dark bottle.

\section{Ethanol extraction method}

This is the traditional extraction method for traditional Chinese medicine according to Miao et al. (2019) with slight modification. The PML was soaked in $70 \%$ ethanol (Guangzhou Hongzhou Chemical Co., Ltd.) and then slowly heated to $70^{\circ} \mathrm{C}$ for $2 \mathrm{~h}$; the liquid was poured through filter paper. The filtered solids were processed through the same extraction steps three times. All the filtration liquids were combined and concentrated into $1 \mathrm{~g} / \mathrm{mL}$ crude extract using a rotary evaporator and stored at $4^{\circ} \mathrm{C}$ in a dark bottle.

\section{Detection of extract components}

Extract volumes of $10 \mathrm{ml}$ of the extract (equivalent to $10 \mathrm{~g}$ of the crude extract) were taken and dried into powder in a vacuum freeze dryer (FD8-5; GOLD SIM International Group). The dry extract was weighed accurately for three times, and the average value was taken as the weight of the extract. The FolinCiocalteu method was used to determine the polyphenols content (Chen et al., 2015), and the anthronesulfuric acid assay was used to determine the content of polysaccharide content in the mulberry leaf extracts (Yu et al., 2019). All tests were performed in triplicate. 


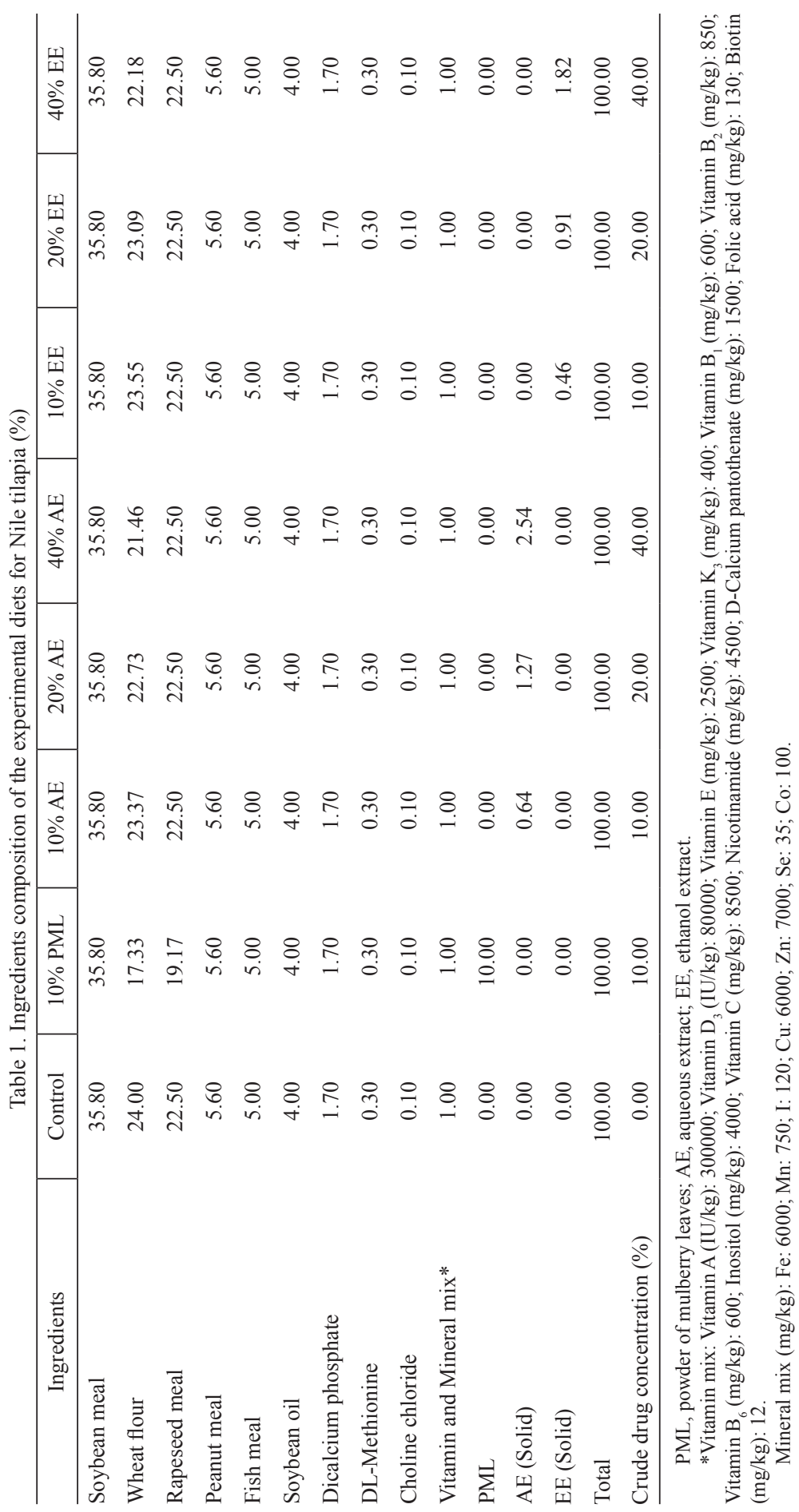




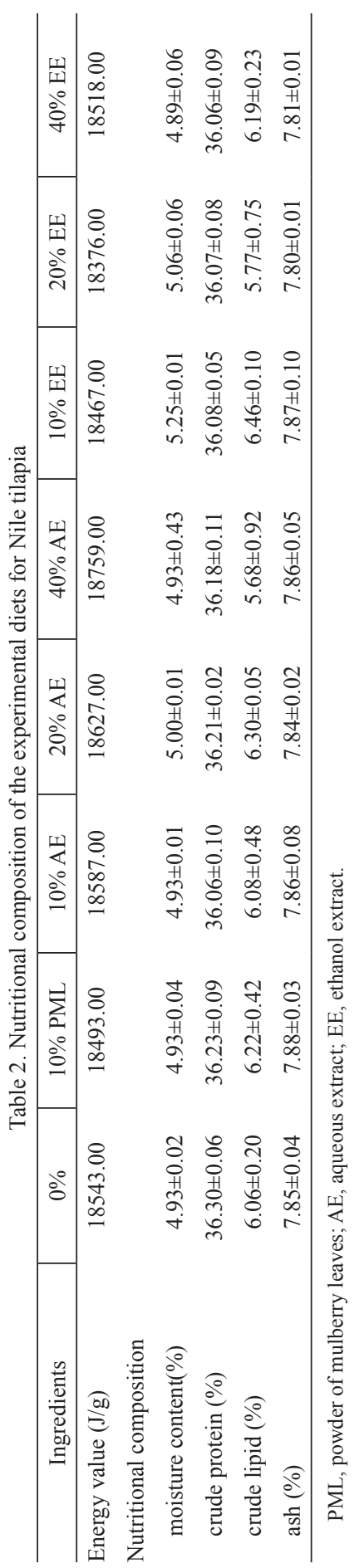




\section{Feed preparation}

The formulation and proximate composition of the test diets prepared for Nile tilapia are shown in Tables 1 and 2. Formulated feed was prepared as a basal diet for the controls or supplemented with $10 \%$ powder of mulberry leaf (PML), three levels of mulberry leaf aqueous extract (AE) $(10 \%, 20 \%$ and 40\%), and three levels of mulberry leaf ethanol extract (EE) $(10 \%, 20 \%$ and $40 \%)$. Feed preparation of these eight diets was done following conventional methods. For tilapia in the $10 \% \mathrm{PML}$ group, PML was directly added to the other dry, raw feed materials for further mixing. For tilapia in the groups fed an extract, AE or EE was first added to water before being added to the other dry, raw feed materials for further mixing. Feed ingredients were weighed and thoroughly mixed in an agitator, followed by addition of oil and water. The wet mash was pelleted to $2.0 \mathrm{~mm}$ in diameter. The resulting pellets were air-dried and then stored at $-20^{\circ} \mathrm{C}$ until use.

\section{Experimental fish and feed conditioning}

A total of 720 Nile tilapia of mean initial weight $8.34 \pm 0.05 \mathrm{~g}$ were obtained from a local provincial breeding farm (GuangDong Province, China). Fish were adaptive cultured in 300-L polyethylene tanks with a recirculating system and water temperature of $27.5 \pm 1{ }^{\circ} \mathrm{C}$, under a $12 \mathrm{~h} \mathrm{light} / 12 \mathrm{~h}$ dark photoperiod, and fed the basal diet for 2 weeks before commencement of the experiment.

\section{Feeding trial and fish sampling}

After the acclimation period, fish were randomly divided into eight groups for the feeding trial: the control, 10\% PML, 10\% AE, 20\% AE, 40\% AE, 10\% EE, 20\% EE and $40 \%$ EE groups, with three replicates per group and 30 fish in each replicate. Fish were fed to satiation twice daily (8:00 and 17:00) for 8 weeks. The body weight and body length of all fish in each group were determined after being euthanized by 3-aminobenzoic acid ethyl ester methanesulfonate (MS-222, 20mg/L), and the spleen, muscle tissue, intestine, and blood samples were collected. Blood was drawn from the caudal vein and centrifuged to coagulate and separate the serum. All the samples were frozen in liquid nitrogen and stored at $-80^{\circ} \mathrm{C}$ until use.

\section{Fish growth performance and survival parameters}

The growth performance and survival parameters of the cultured Nile tilapia were calculated at the end of the feeding trial. All 30 fish in each replicate were individually weighed for calculation of the following indexes:

Survival rate $(\mathrm{SR}, \%)=($ final number of fish $) /($ initial number of fish $) \times 100$.

Weight gain rate $(\mathrm{WGR}, \%)=($ final body weight - initial body weight $) /$ initial body weight $\times 100$.

Specific growth rate $\left(\mathrm{SGR}, \%\right.$ day $\left.^{-1}\right)=(\mathrm{Ln}$ final individual weight - Ln initial individual weight) $/$ (number of days $) \times 100$.

Feed efficiency $($ FE, \% $)=($ weight gain $/$ total feed intake $) \times 100$.

Protein efficiency ratio $(\mathrm{PER})=($ final body weight - initial body weight $) /($ total feed weight $\times$ feed protein content $) \times 100$.

Condition factor $(\mathrm{CF}, \%)=($ body weight, $\mathrm{g}) /($ body length, $\mathrm{cm}) \times 100$. 


\section{Analysis of antioxidant activity and non-specific immunity}

The activities of superoxide dismutase (SOD), catalase (CAT), total antioxidant capacity (T-AOC), acid phosphatase (ACP) and alkaline phosphatase (ALP) were determined in serum, following the specifications of the relevant determination kits provided by the Nanjing Jiancheng Institute of Bioengineering (Nanjing, China).

\section{Immune cytokines quantification by qRT-PCR}

After the feeding trial, the immune cytokines in spleen of Nile tilapia, including interleukin-1 (IL-1), interleukin-2 (IL-2), interleukin-10 $(I L-10)$, interferon- $\gamma(I F N-\gamma)$ and Toll-like receptor 2 (TLR-2), were quantified by qRT-PCR using TaqMan ${ }^{\circledR}$ technology, according to manufacturer's instructions and as previously described (Livak and Schmittgen, 2001). Total RNA was isolated from the samples by using the TRIzol (Invitrogen, USA) reagent according to the manufacturer's protocol. The quality of RNA was examined by gel electrophoresis (1.5\% agarose gel) with an Eppendorf BioPhotometer Model \#6131 (Germany). Sequences of primers for the immune cytokines are given in Table 3. The qRT-PCR was performed with standard protocols on an ABI PRISM $^{\circledR} 7500$ Fast Sequence Detection System. The Ct values were subtracted from the respective $\mathrm{Ct}$ value of the $\beta$-actin control, resulting in the $\Delta \mathrm{Ct}$ value. The largest $\Delta \mathrm{Ct}$ value was arbitrarily used as a constant that was subtracted from all other $\Delta \mathrm{Ct}$ values to determine the $\Delta \Delta \mathrm{Ct}$ value. Fold changes were then generated by calculating $2^{-\Delta \Delta C t}$ (Livak and Schmittgen, 2001). All assays were performed three times.

Table 3. The primer sequences of immune cytokines of Nile tilapia

\begin{tabular}{lll}
\hline \multicolumn{1}{c}{ Item } & \multicolumn{1}{c}{ Primer sequences } & GenBank Acc \\
\hline IL-1-F: & 5' ACCTTCGAAGAATACCATTG & 100707066 (Nile tilapia) \\
IL-1-R: & 5' TCCGCTTAAGAGTTAAAGTG & 100499590 (rainbow trout) \\
IL-2-F: & 5' ATTCAGCAACAAATCCATCT & \\
IL-2-R: & 5' GTAGGCGATCGTAGAATTAG & 100694754 (Nile tilapia) \\
IL-10-F: & 5' ACAGTTCGACATCAACAATC & \\
IL-10-R: & 5' CAGGTACGTCTCAAAGTAGT & \\
IFN- $\gamma$-F: & 5' CGTATTTCCTAGTGACCAGA & 100698762 (Nile tilapia) \\
IFN- $\gamma-R:$ & 5' CGATGTGGTCATTCATCTTG & \\
TLR-2-F: & 5' CGCTATGAGCTTGACTTCTC & \\
TLR-2-R: & 5' GCAGTTTGTAGAAGCGTTTG & 100534414 (Nile tilapia) \\
$\beta-$-actin-F: & 5' TGGTATGGAATCCTGCGGAA & \\
$\beta-$-actin-R: & 5' AGAGAGAGGCCAGGATGGAG & \\
\hline
\end{tabular}

IL, interleukin; IFN, interferon; TLR, Toll-like receptors.

\section{Measurements of meat-quality parameters}

After the 8-week feeding trial, samples of the dorsal muscle of nine fish from each group were collected for measurement of muscle-quality parameters, including $\mathrm{pH}$, the water-holding capacity, percentage cooking loss and amino acid composition. 
The $\mathrm{pH}$ of meat was measured using a $\mathrm{pH}$ meter. Approximately $2.5 \mathrm{~g}$ of finely minced meat from each fish was homogenized for 30 seconds, using an Ultra-Turrax device, in $25 \mathrm{ml}$ of sodium iodoacetate $(5 \mathrm{mM})$ and $18 \mathrm{ml}$ of potassium chloride $(150 \mathrm{mM})$, which was previously equilibrated at $\mathrm{pH} 7$ with the aid of a potassium hydroxide solution $(0.1 \mathrm{~N})$ and of hydrochloric acid $(0.1 \mathrm{~N})$. The $\mathrm{pH}$ of the homogenate sample, which was previously calibrated using buffer solutions at $\mathrm{pH} 4$ and 7 , was determined using a pH meter (Petracci and Baeza, 2011). The test was done in triplicate, with the mean values used for the analysis.

The water-holding capacity (WHC) of meat was determined using the standard filter-paper press technique (Komolka et al., 2020). Three fresh fillets of $5 \pm 0.05 \mathrm{~g}$ from each fish were cut out and each was placed onto separate filter papers. The sample on the filter paper was pressed for 2 min under the constant pressure of $5 \mathrm{~kg}$ during the measurement process. After the pressing, the meat was peeled off the filter paper and weighed again. The difference in weight of the meat was recorded to calculate the water-holding capacity (WHC, expressed as percentage of the initial weight of the sample): WHC $(\%)=$ (initial sample weight - sample weight after pressure) / initial sample weight $\times 100$.

To measure cook loss, three fresh fillets, each $5 \pm 0.05 \mathrm{~g}$, from each fish were cut out and weighed individually, vacuum packaged in a plastic bag, and cooked by immersion in a water bath $\left(80^{\circ} \mathrm{C}\right)$ until their final internal temperature reached $80^{\circ} \mathrm{C}$, following the recommendations of Petracci and Baeza (2011). The cooked samples of meat were cooled under running water for $30 \mathrm{~min}$, and then removed from the bags, blotted dry and weighed. Cook loss was determined by calculating the difference in weight of the samples before and after cooking, expressed as a percentage of the initial weight.

The amino acid composition of the muscle of fish from each group was determined with an automatic amino acid analyzer (Hitachi L-8900, Hitachi (China) Ltd.).

\section{Morphological investigation of intestine samples}

Sections of the fore and middle intestine were excised from nine fish in each group and were then flushed with $0.9 \%$ saline to remove the entire contents. The collected segments of intestine were routinely embedded in paraffin wax blocks, sectioned to $5 \mu \mathrm{m}$ thickness, mounted onto glass slides, and stained with haematoxylin and eosin (H\&E). The morphometric aspects evaluated were the number of intestinal folds, thickness of the muscularis and the villus length. Morphometric measurements were performed on 10 intact and well-oriented villi, and 10 crypts chosen from the duodenum, jejunum and ileum (Qaisrani et al., 2014).

\section{Statistical analyses}

All data are presented as means $\pm \mathrm{SE}$ (standard error). Means were analyzed by one-way analysis of variance (ANOVA) with SPSS 22.0. Significant differences and means were tested using Duncan's test to compare the means of each group. $\mathrm{P}<0.05$ was the chosen significance level. 


\section{Results}

\section{Components of the mulberry leaf extracts}

The solid yield of mulberry leaf and the contents of polysaccharides and polyphenols in the two extracts obtained with the different extraction methods are listed in Table 4. There were some differences in the components between the two extraction methods. The solid yield of mulberry leaf by aqueous extraction was higher than that by ethanol extraction. The content of total polysaccharides was higher in the mulberry leaf aqueous extract (AE) than in the mulberry leaf ethanol extract (EE), while the content of total polyphenols was lower in AE than in EE.

Table 4. Content of polysaccharides and polyphenols in aqueous and ethanol extracts of mulberry leaf $(\mathrm{mg} / \mathrm{g})$

\begin{tabular}{l|c|c|c}
\hline \multicolumn{1}{c|}{ Extraction solvent } & Solid content & Polysaccharides & \multicolumn{1}{c}{ Polyphenols } \\
\hline Double distilled water & $126.97 \pm 1.26$ & $21.96 \pm 1.00$ & $6.10 \pm 0.48$ \\
$70 \%$ ethanol & $90.78 \pm 0.24$ & $14.05 \pm 0.72$ & $13.30 \pm 0.49$ \\
\hline
\end{tabular}

\section{Effects of mulberry leaf extracts on growth performance of Nile tilapia}

The effects of dietary supplementation with AE and EE on the growth performance of cultured Nile tilapia are presented in Table 5. In general, both extracts could increase the growth performance of the Nile tilapia with no significant negative effects on the survival rate $(\mathrm{SR})(\mathrm{P}>0.05)$. The final weight, weight gain rate (WGR) and specific growth rate (SGR) were significantly increased in the $10 \% \mathrm{AE}$, $10 \% \mathrm{EE}$, and $20 \% \mathrm{EE}$ groups when compared with the controls $(\mathrm{P}<0.05)$. The final weight, WGR and SGR showed a decreasing trend in the 10\% PML group though the differences were not significant compared with the controls $(\mathrm{P}>0.05)$; however, these indices were significantly higher in most groups fed an extract as compared with the $10 \%$ PML group $(\mathrm{P}<0.05)$. Feed efficiency ( $\mathrm{FE})$ was significantly decreased in the $20 \% \mathrm{EE}, 40 \% \mathrm{EE}$, and 10\% PML groups compared with the controls $(\mathrm{P}<0.05)$. The protein efficiency ratio (PER) in the 10\% PML group was significantly decreased when compared with fish in the control group and all the extract groups $(\mathrm{P}<0.05)$. The condition factor (CF) was significantly increased in fish of all the extract groups as compared with the controls and the 10\% PML groups. Overall, these results indicated that the extracts of mulberry leaf had subtle, increasing effects on the growth performance of cultured Nile tilapia, and dietary supplementation with either form of the extracts resulted in obviously better growth rates than supplementation with $10 \%$ PML, which had little effect on the growth performance of fish, although no effect on the survival rate. 


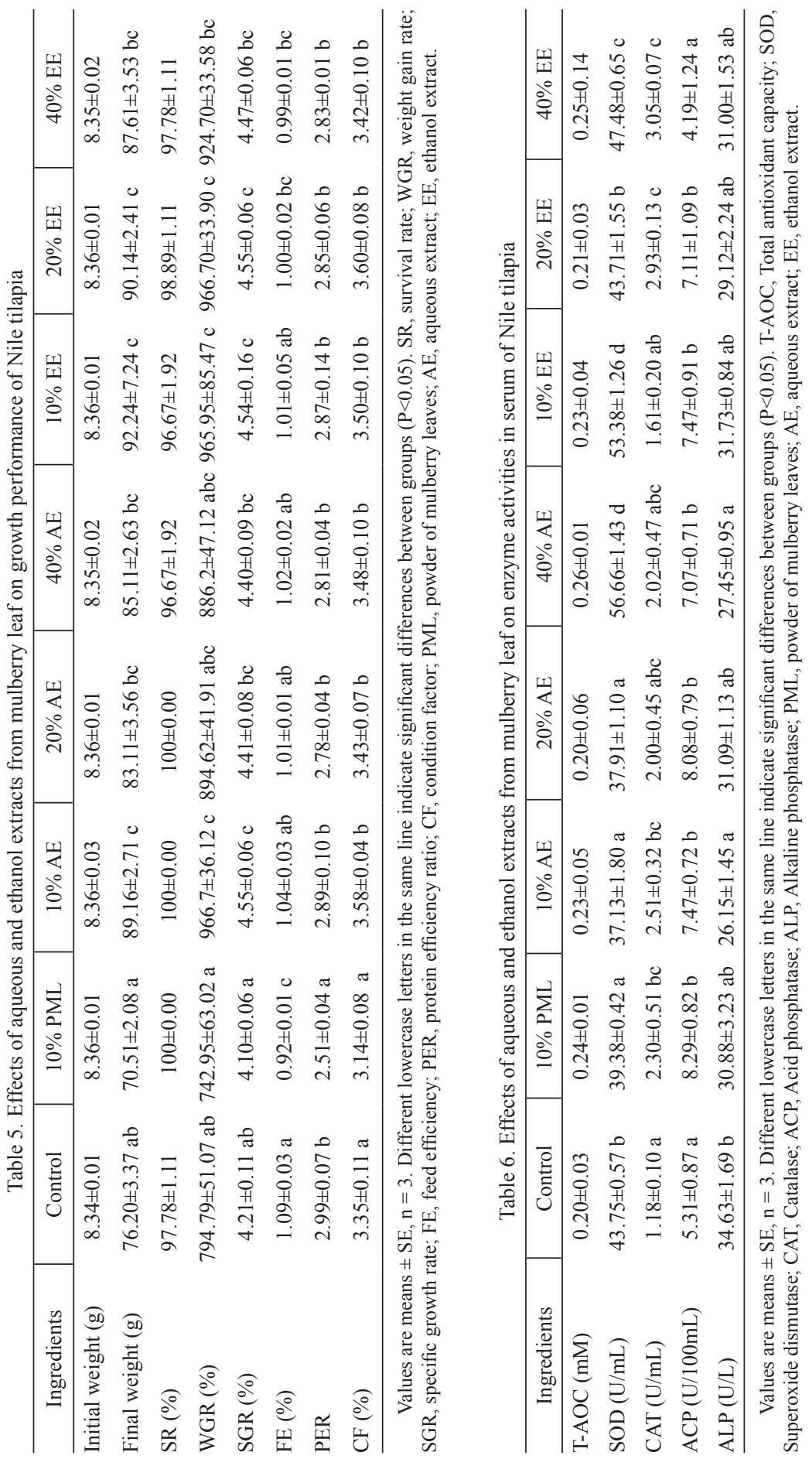




\section{Effects of mulberry leaf extracts on antioxidant activity in Nile tilapia}

The effects of dietary supplementation with $\mathrm{AE}$ and $\mathrm{EE}$ on antioxidant activities in the serums of Nile tilapia are shown in Table 6. The T-AOC in the 10\% PML group and in most of the extract groups showed an increase though the difference was not significant when compared with the control group ( $\mathrm{P}>0.05)$. Compared with the control group, the SOD activities were significantly increased in the $40 \% \mathrm{AE}$, $10 \% \mathrm{EE}$, and $40 \% \mathrm{EE}(\mathrm{P}<0.05)$, but decreased significantly in the $10 \% \mathrm{AE}, 20 \% \mathrm{AE}$ and $10 \%$ PML groups $(\mathrm{P}<0.05)$; the CAT activities were significantly increased in the $10 \%$ PML group as well as in most of the extract groups $(\mathrm{P}<0.05)$. Therefore, it can be concluded that mulberry leaf extract could increase the antioxidant activities in serums of Nile tilapia, and the effects were better with EE than with AE, and the activities were all higher than attained with 10\% PML.

\section{Effects of mulberry leaf extracts on immune responses in Nile tilapia}

The acid phosphatase (ACP) and alkaline phosphatase (ALP) activities were determined to analyze the non-specific immune response in Nile tilapia fed mulberry leaf extracts (Table 6). The ACP activity was significantly increased in the 10\% PML group as well as in all the extract groups other than the the 40\% EE group $(\mathrm{P}<0.05)$, in which it was similar to that of the 10\% PML group. The ALP activity in the $10 \%$ AE and $40 \%$ AE groups was significantly lower than that of the controls $(\mathrm{P}<0.05)$, whereas there were no significant differences between the other groups and the controls $(\mathrm{P}>0.05)$.
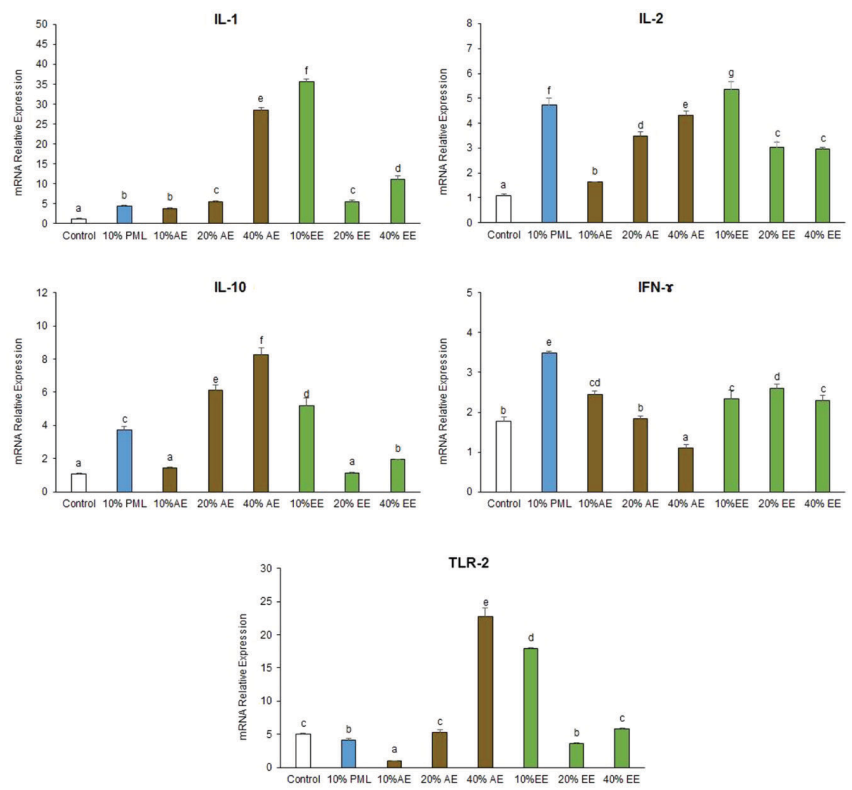

Figure 1. Effects of mulberry leaf extracts on immune activity in spleen of Nile tilapia. Results represent at least three independent assays compared with Duncan's test. Values are means $\pm \mathrm{SE}$ of triplicate groups. Different lowercase letters denote significant differences $(\mathrm{P}<0.05)$ among treatments with powder of mulberry leaf (PML), mulberry leaf aqueous extract (AE), and mulberry leaf ethanol extract (EE). $I L-1$, Interleukin-1; IL-2, Interleukin-2; IL-10, Interleukin-10; IFN- $\gamma$, Interferon- $\gamma ; T L R-2$, Toll-like Receptor 2 


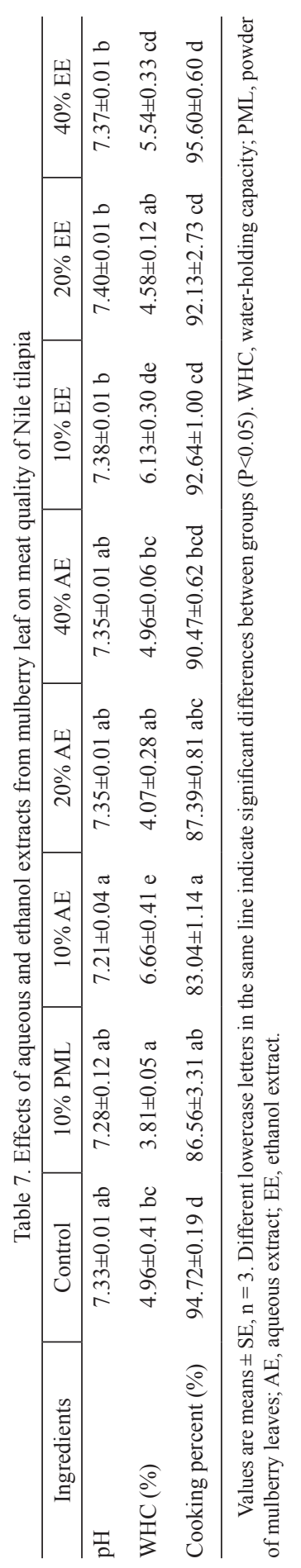




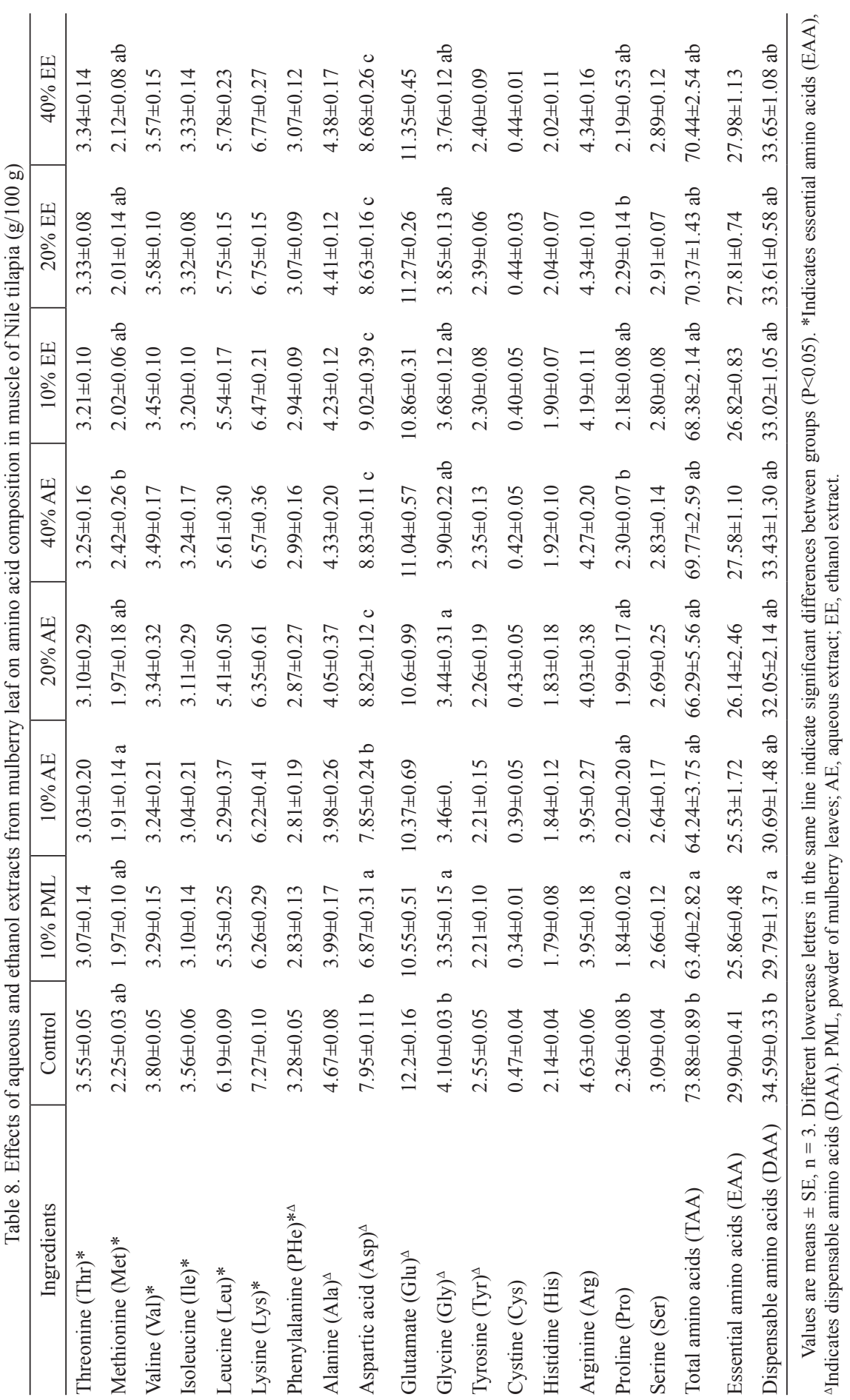


The expressions of immune cytokines in spleen as a reflection of the immune function in the Nile tilapia were examined (Figure 1). The expressions of $I L-1, I L$ 2 , and $I L-10$ were significantly increased in most of the extract groups as well as in the $10 \%$ PML group when compared with the controls $(\mathrm{P}<0.05)$, and the effects in fish of the extract groups were better than seen in the 10\% PML group for $I L-1$ and $I L-10$. Several of the extract groups and the 10\% PML showed increased expression of IFN- $\gamma$. For TLR-2, some of the extract groups and the 10\% PML showed reduced effects, whereas in the $40 \% \mathrm{AE}$ and $10 \% \mathrm{EE}$ groups the expression of TLR-2 was significantly increased $(\mathrm{P}<0.05)$. The expressions of $I L-1, I L-2, I L-10$, and $T L R-2$ in the $10 \%$ EE group were significantly increased as compared with the $10 \% \mathrm{PML}$ and $10 \%$ AE groups $(\mathrm{P}<0.05)$. The results for most treatment groups showed that the mulberry leaf extract could improve the expressions of immune cytokines, and the effect was better than attained with PML.

\section{Effects of mulberry leaf extract on meat quality of Nile tilapia}

The meat qualities of each treatment group are summarized in Table 7. The $\mathrm{pH}$ of muscle across the groups was neutral to slightly alkaline, although there were no significant differences among the extract groups, 10\% PML group and the controls $(\mathrm{P}>0.05)$.

The water-holding capacity (WHC) was significantly lower in the $10 \% \mathrm{PML}$ group than that in most of the extract groups of mulberry leaf and the controls $(\mathrm{P}<0.05)$, but it was significantly higher in the $10 \% \mathrm{AE}$ and $10 \% \mathrm{EE}$ groups than that in the controls $(\mathrm{P}<0.05)$. The percentage cook loss in groups $10 \% \mathrm{AE}, 20 \% \mathrm{AE}$ and $10 \%$ PML was significantly lower than that in the control group $(\mathrm{P}<0.05)$, but no significant changes were found for other groups when compared with the controls $(\mathrm{P}>0.05)$. The three indicators of meat quality showed that mulberry leaf extract could improve meat quality of Nile tilapia, with the effects of dietary supplementation with EE better than with AE. In contrast, fish fed the 10\% PML diet exhibited poorer meat quality.

The results of $\mathrm{AE}$ and $\mathrm{EE}$ supplementation on the amino acid composition in Nile tilapia muscle are listed in Table 8, and no significant difference in the content of essential amino acids (EAA) was found in each group ( $\mathrm{P}>0.05)$. The contents of total amino acids (TAA), the dispensable amino acids (DAA) and proline were significantly lower in the $10 \%$ PML group than in control group $(\mathrm{P}<0.05)$, but these contents did not show a significant change in the other groups $(\mathrm{P}>0.05)$. Most extract groups significantly increased the content of aspartic acid (Asp) $(\mathrm{P}<0.05)$, whereas the $10 \%$ PML group significantly reduced the Asp content $(\mathrm{P}<0.05)$. Therefore, the results indicate that the mulberry leaf extract not only had no negative effect on the contents of EAA and TAA in Nile tilapia muscle, but also could increase the content of some DAA, such as Asp, while the 10\% PML diet reduced the content of EAA, TAA and DAA in the fish.

\section{Effects of mulberry leaf extract on intestinal morphology in Nile tilapia}

The intestinal morphology of the Nile tilapia in each treatment group is presented in Figure 2 and Table 9. The assessments of the fore intestine of the fish showed that 
the $10 \% \mathrm{AE}, 10 \% \mathrm{EE}$ and $10 \%$ PML groups presented no changes in regard to the three intestinal morphology parameters, including the number of intestinal folds, thickness of the muscularis, and villi lengths. Assessments of the middle intestine of the $10 \% \mathrm{AE}$ and $10 \% \mathrm{EE}$ groups revealed significantly increased villus length compared with the controls and the 10\% PML group $(\mathrm{P}<0.05)$; however, the $10 \%$ PML group showed significantly decreased muscularis thickness compared with the control group and the $10 \% \mathrm{AE}$ and $10 \%$ EE groups $(\mathrm{P}<0.05)$. In summary, the mulberry leaf extract treatment had positive effects on the intestinal morphology of Nile tilapia, to some extent, in contrast to the $10 \%$ PML treatment.

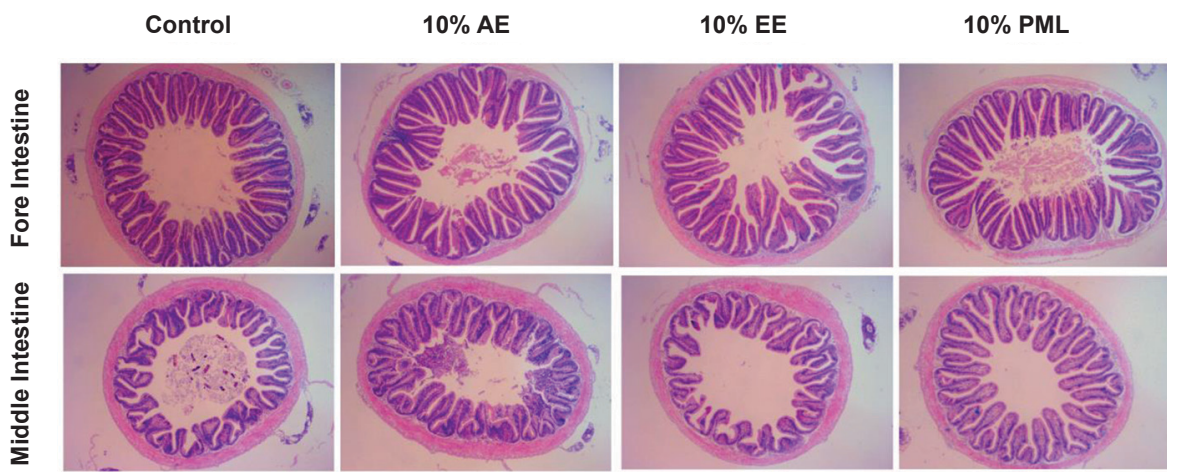

Figure 2. Histopathology of intestine sections sampled from Nile tilapia that were fed diets with mulberry leaf extract. Results represent assessments of the histopathological photographs of H\&E-stained sections of the fore intestine and middle intestine, in the control, $10 \% \mathrm{AE}, 10 \% \mathrm{EE}$, and $10 \% \mathrm{PML}$ groups (40× magnification). AE, aqueous extract; EE, ethanol extract; PML, powder of mulberry leaf

Table 9. Effects of aqueous and ethanol extracts from mulberry leaf on intestinal morphology in Nile tilapia

\begin{tabular}{|c|c|c|c|c|}
\hline Ingredients & Control & $10 \% \mathrm{PML}$ & $10 \% \mathrm{AE}$ & $10 \% \mathrm{EE}$ \\
\hline \multicolumn{5}{|l|}{ Fore intestine } \\
\hline number of folds & $52.00 \pm 0.58$ & $50.33 \pm 2.60$ & $50.67 \pm 1.76$ & $52.67 \pm 2.03$ \\
\hline muscularis thickness $(\mu \mathrm{m})$ & $113.95 \pm 5.94$ & $118.90 \pm 5.33$ & $119.42 \pm 1.71$ & $122.44 \pm 2.27$ \\
\hline villus length $(\mu \mathrm{m})$ & $543.46 \pm 23.93$ & $536.23 \pm 16.06$ & $541.56 \pm 9.87$ & $539.48 \pm 13.83$ \\
\hline \multicolumn{5}{|l|}{ Middle intestine } \\
\hline number of folds & $37.33 \pm 2.40$ & $35.67 \pm 2.73$ & $37.00 \pm 1.53$ & $39.67 \pm 2.03$ \\
\hline muscularis thickness $(\mu \mathrm{m})$ & $133.41 \pm 1.75 b$ & $104.09 \pm 4.98 \mathrm{a}$ & $134.79 \pm 0.79 b$ & $134.19 \pm 1.09 \mathrm{~b}$ \\
\hline villus length $(\mu \mathrm{m})$ & $226.63 \pm 4.97 \mathrm{a}$ & $215.35 \pm 15.15 \mathrm{a}$ & $258.18 \pm 4.17 \mathrm{~b}$ & $256.03 \pm 5.50 \mathrm{~b}$ \\
\hline
\end{tabular}

Values are means $\pm \mathrm{SE}, \mathrm{n}=3$. Different lowercase letters in the same line indicate significant differences between groups $(\mathrm{P}<0.05)$. $\mathrm{PML}$, powder of mulberry leaves; $\mathrm{AE}$, aqueous extract; $\mathrm{EE}$, ethanol extract. 


\section{Discussion}

Mulberry leaf can be used as a potential fishmeal replacement in formulated feeds for fish (Kaviraj et al., 2013; Mondal et al., 2015; Sheikhlar et al., 2014). Besides being a good protein source in animal culture, mulberry leaf is also a traditional Chinese medicine. More and more countries have recognized the negative effects of antibiotics in aquaculture and animal agriculture; hence, the importance of antibiotic-free feeding has been recently realized. Chinese herbal medicine is regarded a prime candidate to achieve antibiotic-free feeding among the many alternatives to antibiotics.

Polysaccharides and polyphenols are two main components among the many bioactive compounds in mulberry leaf. The polysaccharides in mulberry leaves have attracted increasing attention based on their multiple biological activities, such as antidiabetic, anti-tumor, anti-inflammatory, immunostimulatory effects and antioxidant activities, etc (He et al., 2018). Polyphenols have been extensively investigated with regard to their antioxidant, anti-inflammatory, and immunomodulant properties in relation to several chronic inflammatory conditions (Oliviero et al., 2018) and polyphenolic compounds are often used as potential chemical markers for the antioxidant properties of herbs (Ganzon et al., 2018).

In the present study, two traditional extraction methods for the Chinese herbal medicines were used and compared to evaluate the effects of mulberry leaf extract as a dietary supplement for Nile tilapia. Accordingly, the contents of polysaccharides and polyphenols in the different mulberry leaf extracts were determined. We also identified differences in the components of the extracts obtained by either the aqueous or ethanol extraction methods. The content of total polysaccharides was likewise higher in the aqueous extract than in the ethanol extract, whereas the content of total polyphenols was lower in the aqueous extract than in ethanol extract. Our subsequent investigation into the effects of dietary supplementation with mulberry leaf extract on growth, immune response and antioxidant functions in cultured Nile tilapia showed differences between fish fed the aqueous extract and ethanol extract, which here were mainly related to the differences in the composition and contents of the bioactive substances, as obtained by the different extraction methods. With modernization of the processing methods for traditional Chinese medicines, more and more techniques such as ultrasonic, microwave supercritical fluid extraction and reflux extraction have been applied to extract the effective components (Hou et al., 2010; Zhou et al., 2017). However, for polysaccharide extraction, the hot-water extraction technique is still the main and classic method owing to its convenience, low cost and high extraction yield (Passos and Coimbra, 2013; Wei et al., 2010), though the existing water extraction method takes more time and requires several extraction cycles. Moreover, further study is needed to determine whether some other active compounds remain in the extract.

Previous researchers have reported on the effects of mulberry leaf and fermented mulberry leaf meal as a fishmeal replacement in feeds for fish. However, there have been few studies about the effects of mulberry leaf extracts as a medicine or functional feed additive in fish. Our study investigated the effects of mulberry leaf 
extract on growth, immune response, and antioxidant functions in Nile tilapia and compared this form of dietary supplementation with mulberry leaf powder $(10 \%)$ added directly to the feed. The results showed that treatment with the extracts from mulberry leaf significantly improved the growth performance, increased the antioxidant activities and acid phosphatase activity in serums, increased the expressions of immune cytokines, improved the meat quality, and benefited intestinal morphology when compared with the control fish. Moreover, the mulberry leaf extracts from mulberry leaf showed better effects than use of $10 \%$ PML in most of the above aspects. Therefore, our research indicates that mulberry leaf extract is more suitable as a feed additive than mulberry leaf powder for enhancing the growth and health of cultured Nile tilapia.

Mulberry leaf is an important ingredient in some traditional Chinese medicinal formulas and is considered to have high nutritional value and antioxidant activity, which could be developed for use in foods benefiting human health (Liang et al., 2012). The mulberry leaf extracts were also found to increase the antioxidant activities in serums of Nile tilapia. The effect of ethanol extract was slightly better than that of aqueous extract. Our findings are similar to those of Wang et al. (2012) who reported that ethanolic extracts from mulberry leaves, stems and fruit showed higher contents of total phenolics than aqueous extracts, and the antioxidant activities of ethanolic extracts from mulberry leaf were stronger than from aqueous extracts. Polyphenols, the most commonly found chemical compounds in herbal beverages and foods, are reported to influence reactive oxygen species regulation, confer neuroprotective effects, and impact cell signal transduction (Ebrahimi and Schluesener, 2012). Mulberry leaves are reported to contain abundant varieties and quantities of polyphenols which have extensive functions in antioxidant and free-radical scavenging in vitro (Choi et al., 2013; Zou et al., 2012). However, there is cumulative evidence to suggest that polysaccharides of mulberry possess antioxidant activity (He et al., 2018; Liu et al., 2017; Ma et al., 2018). The antioxidant activities in the Nile tilapia as a consequence of the mulberry leaf extract treatments should be related mainly to the polysaccharides and polyphenols in the leaves. However, it is not yet clear which components of the extracts play a specific role in the antioxidant functions, and the mechanisms of action of these ingredients would need to be extensively researched.

The immunomodulatory activities of mulberry leaf have been described in many reports. In vitro, the water extract of Mori folium (WEMF) showed a potential to modulate the immune function by regulating immunological parameters in the murine macrophage cell line RAW264.7, and the release and expression of cytokines, such as $T N F-\alpha, I L-1 \beta, I L-6$, and $I L-10$, were also significantly increased in response to treatment with WEMF. Moreover, WEMF promoted differentiation of the RAW264.7 macrophagic cells and the resulting phagocytes' activity (Kwon et al., 2016). Zhao et al. (2019) found that when added to diets for weanling pigs, the polysaccharides of mulberry leaf increased the serum levels of immunoglobulin G (IgG), $I L-1 \beta, I L-2, I L-8$, and $I F N-\gamma$, which improved the animals' metabolisms and immune functions; furthermore, were superior in a WEMF low-dose group as compared with both a WEMF high-dose group and an antibiotic group. Mulberry leaf polysaccha- 
ride was also found to stimulate spleen lymphocyte proliferation in mice in vitro; significantly improve antibodies to the Newcastle disease in serum titer as well as the concentrations of $I L-2, I F N-\gamma$, and secretory immunoglobulin A (sIgA) in tracheal and jejunal wash fluids; and increase the numbers of immunoglobulin A-positive $\left(\operatorname{Ig} \mathrm{A}^{+}\right)$cells in cecal tonsils in chickens (Chen et al., 2019). In the present study, the expressions of $I L-1, I L-2, I L-10, I F N-\gamma$ and $T L R-2$ were similarly increased by feed supplementation with the mulberry leaf extracts. Our results therefore suggest that the active components of mulberry leaf could significantly enhance immune activity in a fish and might be a potential immunopotentiating drug candidate.

The effects of the extracts on Nile tilapia differed with their proportion in the feed. The 10\% AE and 10\% EE diets showed the best effects on the fish. These treatments both significantly increased the SGR, the CAT activity, and villus length in the middle intestine as compared with the controls and the $10 \%$ PML group. In addition, the $10 \%$ EE diet had a better effect on the expressions of $I L-1, I L-2, I L-10$, $T N F-\alpha$, and TLR-2 than the other diets. Comprehensive consideration of the growth performance, antioxidant activities and immune response indicators suggests that $10 \% \mathrm{EE}$ added to the diet is recommended for enhancing the growth rate and health status of Nile tilapia.

\section{Conclusions}

This study prepared mulberry leaf extracts using two traditional methods, namely aqueous extraction and ethanol extraction, then identified the effects in Nile tilapia fed diets with different levels of the supplementation. Fish fed an extract obtained with either method displayed better growth performance, increased antioxidant activities and ACP in serum, increased expression of immune cytokines, and improved intestinal morphology compared with controls and fish given 10\% mulberry leaf powder supplementation. In conclusion, $10 \%$ mulberry leaf ethanol extract added to a formulated feed is recommended for enhancing the growth rate and health status of cultured Nile tilapia.

\section{Acknowledgments}

This work was supported by grants from the National Natural Science Foundation of China (No.31302213, No.31001117), the Science and Technology plan projects of Guangdong province (No.2018LM2159, No.2015A020209103), and the Central Public-interest Scientific Institution Basal Research Fund, South China Sea Fisheries Research Institute, CAFS (No.2016TS17).

\section{References}

Ali S., Saha S., Kaviraj A. (2020). Fermented mulberry leaf meal as fishmeal replacer in the formulation of feed for carp Labeo rohita and catfish Heteropneustes fossilis - optimization by mathematical programming. Trop. Anim. Health Prod., 52: 839-849.

C a i M., Mu L., Wang Z.L., Li u J.Y., L i u T.L., Wa n a p a t M., H u a g g B.Z. (2019). Assessment of mulberry leaf as a potential feed supplement for animal feeding in P.R. China. Asian-Australas. J. Anim. Sci., 32: 1145-1152. 
Chan E.W., Lye P.Y., Wong S.K. (2016). Phytochemistry, pharmacology, and clinical trials of Morus alba. Chin. J. Nat. Med., 14: 17-30.

Chen C., Moh a mad R.U., S a i ki m F.H., Mahyudin A., Mohd N.N. (2021). Morus alba L. plant: bioactive compounds and potential as a functional food ingredient. Foods, 10(3).

Chen L.Y., Cheng C.W., Liang J.Y. (2015). Effect of esterification condensation on the FolinCiocalteu method for the quantitative measurement of total phenols. Food Chem., 170: 10-15.

Chen X., Sheng Z., Qi u S., Yang H., Ji a J., Wang J., Ji ang C. (2019). Purification, characterization and in vitro and in vivo immune enhancement of polysaccharides from mulberry leaves. PLoS One, 14(1): e208611.

Cho i J., K ang H.J., K i m S.Z., K w on T.O., J e on g S.I., J a n g S.I. (2013). Antioxidant effect of astragalin isolated from the leaves of Morus alba L. against free radical-induced oxidative hemolysis of human red blood cells. Arch. Pharm. Res., 36: 912-917.

Ebrah i m i A., S c h 1 u e s e n e r H. (2012). Natural polyphenols against neurodegenerative disorders: potentials and pitfalls. Ageing. Res. Rev., 11: 329-345.

E 1 - S a y y a d H.I. (2015). Cholesterol overload impairing cerebellar function: the promise of natural products. Nutrition, 31: 621-630.

F u Y., Zhang Q., X u D.H., Xi a H., C a i X., Wang B., Li ang J. (2014). Parasiticidal effects of Morus alba root bark extracts against Ichthyophthirius multifiliis infecting grass carp. Dis. Aquat. Organ., 108: 129-136.

G a n z o n J.G., C h e n L.G., W a n g C.C. (2018). 4-O-caffeoylquinic acid as an antioxidant marker for mulberry leaves rich in phenolic compounds. J. Food Drug. Anal., 26: 985-993.

H a o J.Y., Wan Y., Ya o X.H., Zha o W.G., Hu R.Z., Chen C., Li L., Zhang D.Y., Wu G.H. (2018). Effect of different planting areas on the chemical compositions and hypoglycemic and antioxidant activities of mulberry leaf extracts in Southern China. PLoS One, 13(6): e198072.

He X., F ang J., R u a Y., Wang X., Sun Y., Wu N., Zh a o Z., Chang Y., Ning N., Guo H., $\mathrm{H}$ u a n g L. (2018). Structures, bioactivities and future prospective of polysaccharides from Morus alba (white mulberry): A review. Food Chem., 245: 899-910.

Hou J., He S., Ling M., Li W., Dong R., Pan Y., Zheng Y. (2010). A method of extracting ginsenosides from Panax ginseng by pulsed electric field. J. Sep. Sci., 33: 2707-2713.

K a n d y lis K., Ha d j i g e org i o u I., H a ri z a n is P. (2009). The nutritive value of mulberry leaves (Morus alba) as a feed supplement for sheep. Trop. Anim. Health Prod., 41: 17-24.

Kaviraj A., Mond a 1 K., M u kho pa dh y a y P.K., T u r ch in i G.M. (2013). Impact of fermented mulberry leaf and fish offal in diet formulation of Indian major carp (Labeo rohita). Proc. Zoological Society (Calcutta), 66: 64-73.

K o molka K., B o chert R., Franz G.P., K a y a Y., P fuhl R., Grunow B. (2020). Determination and comparison of physical meat quality parameters of Percidae and Salmonidae in aquaculture. Foods, 9(4).

K o ng L., Yang C., D ong L., D i a o Q., S i B., M a J., T u Y. (2019). Rumen fermentation characteristics in pre- and post-weaning calves upon feeding with mulberry leaf flavonoids and Candida tropicalis individually or in combination as a supplement. Animals, 9: 990.

K w o n D.H., Ch e o n J.M., Ch o i E.O., J e ong J.W., L e e K.W., K i m K.Y., K i m S.G., K i m S., Hong S.H., Park C., Hwang H.J., Choi Y.H. (2016). The immunomodulatory activity of Mori folium, the leaf of Morus alba L., in RAW 264.7 macrophages in vitro. J. Cancer Prev., 21: $144-151$.

K w o n D.H., J e ong J.W., C h o i E.O., L e e H.W., L e e K.W., K i m K.Y., K i m S.G., H o n g S.H., K im G.Y., P a rk C., Hw ang H.J., S on C.G., Choi Y.H. (2017). Inhibitory effects on the production of inflammatory mediators and reactive oxygen species by Mori folium in lipopolysaccharide-stimulated macrophages and zebrafish. An. Acad. Bras. Cienc., 89: 661-674.

Li Y., Z h ang X., Li an g C., H u J., Yu Z. (2018). Safety evaluation of mulberry leaf extract: Acute, subacute toxicity and genotoxicity studies. Regul. Toxicol. Pharmacol., 95: 220-226.

L i a n g J.H., F u Y.W., Z h a n g Q.Z., X u D.H., Wang B., L in D.J. (2015). Identification and effect of two flavonoids from root bark of Morus alba against Ichthyophthirius multifiliis in grass carp. J. Agric. Food Chem., 63: 1452-1459.

Liang L., Wu X., Zhu M., Zhao W., Li F., Z ou Y., Yang L. (2012). Chemical composition, nutritional value, and antioxidant activities of eight mulberry cultivars from China. Pharmacon. Mag., 8: 215-224. 
L in W.C., L e e M.T., Chang S.C., Chang Y.L., Sh ih C.H., Yu B., L e e T.T. (2017). Effects of mulberry leaves on production performance and the potential modulation of antioxidative status in laying hens. Poultry Sci., 96: 1191-1203.

L i u C.G., M a Y.P., Z h a n g X.J. (2017). Effects of mulberry leaf polysaccharide on oxidative stress in pancreatic beta-cells of type 2 diabetic rats. Eur. Rev. Med. Pharmacol. Sci., 21: 2482-2488.

L i u Y., Li Y., Peng Y., He J., Xia o D., Chen C., Li F., Huang R., Yin Y. (2019 a). Dietary mulberry leaf powder affects growth performance, carcass traits and meat quality in finishing pigs. J. Anim. Physiol. Anim. Nutr. (Berlin), 103: 1934-1945.

Livak K.J., S chmittgen T.D. (2001). Analysis of relative gene expression data using real-time quantitative PCR and the 2(-Delta Delta C(T)) Method. Methods, 25: 402-408.

M a Q., S a n th a n a m R.K., X u e Z., Gu o Q., G a o X., Ch e n H. (2018). Effect of different drying methods on the physicochemical properties and antioxidant activities of mulberry leaves polysaccharides. Int. J. Biol. Macromol., 119: 1137-1143.

Mahmoud E.A., E1-Sayed B.M., Mahsoub Y.H., E1-Murr A., Neamat-Allah A. (2020). Effect of Chlorella vulgaris enriched diet on growth performance, hemato-immunological responses, antioxidant and transcriptomics profile disorders caused by deltamethrin toxicity in Nile tilapia (Oreochromis niloticus). Fish Shellfish Immunol., 102: 422-429.

Mi a o W.G., Tang C., Ye Y., Qu in n R.J., Feng Y. (2019). Traditional Chinese medicine extraction method by ethanol delivers drug-like molecules. Chin. J. Nat. Med., 17: 713-720.

Mondal K., Kaviraj A., Mukhopadhy y P.K. (2015). Growth performance of Indian minor carp Labeo bata fed varying inclusions of fermented fish-offal and mulberry leaf meal based-diets. Iran. J. Fish. Sci., 14: 567-582.

Ne a m a t- A 11 a h A.N.F., E 1 - M u r r A.E.I., A bd E 1- H a k i m Y. (2019). Dietary supplementation with low molecular weight sodium alginate improves growth, haematology, immune reactions and resistance against Aeromonas hydrophila in Clarias gariepinus. Aquac. Res., 50: 1547-1556.

Ne a mat-A 11 ah A.N.F., Abd El Hakim Y., Mahmoud E.A. (2020). Alleviating effects of $\beta$-glucan in Oreochromis niloticus on growth performance, immune reactions, antioxidant, transcriptomics disorders and resistance to Aeromonas sobria caused by atrazine. Aquac. Res., 51: 1801-1812.

Ne a mat-A 11 ah A.N.F., Mahs oub Y.H., Mahmoud E.A. (2021 a). The potential benefits of dietary $\beta$-glucan against growth retardation, immunosuppression, oxidative stress and expression of related genes and susceptibility to Aeromonas hydrophila challenge in Oreochromis niloticus induced by herbicide pendimethalin. Aquac. Res., 52: 518-528.

N e a m a t - A 11 ah A., Mahmoud E.A., Mah s o u b Y. (2021 b). Effects of dietary white mulberry leaves on hemato-biochemical alterations, immunosuppression and oxidative stress induced by Aeromonas hydrophila in Oreochromis niloticus. Fish Shellfish Immunol., 108: 147-156.

Olivi ero F., S c a n u A., Za mudi o- Cuevas Y., Punzi L., S p in e 11 a P. (2018). Anti-inflammatory effects of polyphenols in arthritis. J. Sci. Food. Agric., 98: 1653-1659.

P a s s o s C.P., C o i m b r a M.A. (2013). Microwave superheated water extraction of polysaccharides from spent coffee grounds. Carbohydr. Polym., 94: 626-633.

P etra c c i M., B a e z a E. (2011). Harmonization of methodologies for the assessment of poultry meat quality features. World Poultry Sci. J., 67: 417-418.

Q a is ran i S.N., Moquet P.C., van Krimpen M.M., Kwakkel R.P., Verstegen M.W., H e n d riks W.H. (2014). Protein source and dietary structure influence growth performance, gut morphology, and hindgut fermentation characteristics in broilers. Poultry Sci., 93: 3053-3064.

She ikhlar A., A 1 i mon A.R., D a u d H., S a d C.R., Webster C.D., Meng G.Y., Ebra h i m i M. (2014). White mulberry (Morus alba) foliage methanolic extract can alleviate Aeromonas hydrophila infection in African catfish (Clarias gariepinus). Sci. World J., 2014: 592709.

Sh e i kh l ar A., G o h Y.M., A 1 i m o n R., E brah i m i M. (2017). Antioxidative effects of mulberry foliage extract in African catfish diet. Aquac. Res., 48: 4409-4419.

Wang W., Zu Y., F u Y., Efferth T. (2012). In vitro antioxidant and antimicrobial activity of extracts from Morus alba L. leaves, stems and fruits. Am. J. Chin. Med., 40: 349-356.

Wei X., Chen M., Xia o J., Liu Y., Yu L., Zhang H., Wang Y. (2010). Composition and bioactivity of tea flower polysaccharides obtained by different methods. Carbohydr. Polym., 79: 418-422. 
Xi ong X., Li H., Q i u N., S u L., H u ang Z., S ong L., Wang J. (2020). Bioconcentration and depuration of cadmium in the selected tissues of rare minnow (Gobiocypris rarus) and the effect of dietary mulberry leaf supplementation on depuration. Environ. Toxicol. Pharmacol., 73: 103278.

Yu Y., Ye H., Wu D., Shi H., Zhou X. (2019). Chemoenzymatic quantification for monitoring unpurified polysaccharide in rich medium. Appl. Microbiol. Biotechnol., 103: 7635-7645.

Zha o X., Yang R., B i Y., Bilal M., Kuang Z., I qbal H., L u o Q. (2019). Effects of dietary supplementation with mulberry (Morus alba L.) leaf polysaccharides on immune parameters of weanling pigs. Animals (Basel), 10(1).

Z h o u J., Yu a n X., Li L., Z h a n g T., W a n g B. (2017). Comparison of different methods for extraction of Cinnamomi ramulus: yield, chemical composition and in vitro antiviral activities. Nat. Prod. Res., 31: 2909-2913.

Zou Y., Li a o S., Shen W., Liu F., Tang C., Chen C.Y., Sun Y. (2012). Phenolics and antioxidant activity of mulberry leaves depend on cultivar and harvest month in Southern China. Int. J. Mol. Sci., 13: 16544-16553.

Received: 30 III 2021

Accepted: 20 V 2021 